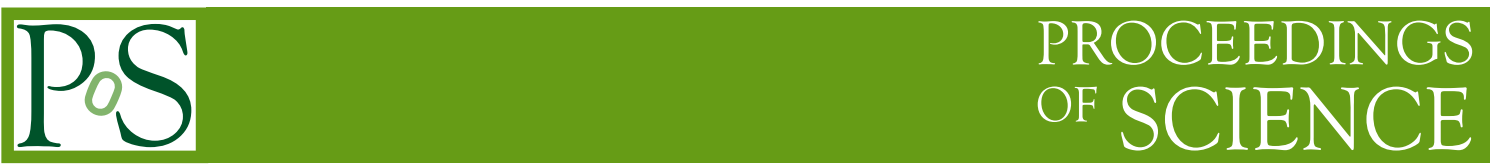

\title{
Gravitational Scattering via Twistor Theory
}

\section{Tim Adamo*}

Dep. of Applied Mathematics and Theoretical Physics, Univ. of Cambridge

E-mail: t.adamo@damtp.cam.ac.uk

Scattering amplitudes are among the most natural-and important-observables of any field theory in an asymptotically flat space-time, including any theory of quantum gravity. Recently, gravitational scattering amplitudes have been shown to posess remarkably compact expressions which are un-expected from the perspective of traditional Lagrangian-based perturbation theory (c.f., [1]). We will discuss how some of these formulae arise from working with an action principal not on space-time, but rather in twistor space, an auxiliary complex three-manifold [2]. This narrative exploits an on-shell equivalence between conformal gravity and general relativity in asymptotically de Sitter manifolds [3], and also leads to new expressions for analogues of scattering amplitudes in space-times with a positive cosmological constant.

\section{References}

[1] F. Cachazo \& D. Skinner, Gravity from Rational Curves in Twistor Space, Phys.Rev.Lett., 110 (2013) 161301 arXiv:1207.0741.

[2] T. Adamo \& L. Mason, Conformal and Einstein gravity from twistor actions, Class.Quant.Grav., 31 (2014) 045014 arXiv:1307.5043.

[3] J. Maldacena, Einstein gravity from conformal gravity, (2011) arXiv:1105.5632.

Frontiers of Fundamental Physics 14 - FFP14,

15-18 July 2014

Aix Marseille University (AMU) Saint-Charles Campus, Marseille

\footnotetext{
* Speaker.
} 